\title{
Promoción del bienestar psicológico en la secundaria: una experiencia piloto
}

\section{Promotion of psychological well-being in secondary school: a pilot experience}

Diego García-Álvarez ${ }^{1}$ M.Sc, María José-Soler², M.Sc, Lourdes Achard-Braga³ , M.Sc.

\begin{abstract}
${ }^{1}$ Centro de Investigación y Experimentación Pedagógica-CIEP, Uruguay. Magíster Scientiarium en Psicología Educacional, Psicólogo y Licenciado en Educación. ${ }^{2}$ Asociación Jóvenes Fuertes, Montevideo, Uruguay. Doctoranda en Psicología, Posgrado en Terapia Cognitivo-Comportamental, Posgrado en Terapia Familiar sistémica, Psicóloga. ${ }^{3}$ Magíster en Enseñanza de Idioma Inglés, Posgrado en Pensamiento Crítico, Posgrado en Evaluación, Posgrado en Educación Especial. Asociación Jóvenes Fuertes, Montevideo, Uruguay. *Correspondencia: diegogarcia_123@hotmail.com.
\end{abstract}

\section{RESUMEN}

Con la finalidad de la promoción del bienestar psicológico en centros educativos, se diseñó una experiencia psicoeducativa basada en el cultivo de las fortalezas del carácter para estudiantes de educación secundaria. La investigación contó con los objetivos de: determinar el nivel de bienestar psicológico en los adolescentes de secundaria; analizar las relaciones entre bienestar psicológico y las fortalezas del carácter; establecer si existen diferencias de género y de acuerdo con el tipo de centro educativo con respecto al bienestar psicológico - sus dimensiones- y las fortalezas del carácter en los adolescentes de secundaria. Metodológicamente se ajusta a una investigación descriptiva con diseño transversal y correlacional a través de la recolección de datos por medio de medidas psicométricas con adecuados índices de confiabilidad y validez. Como resultados se tienen niveles adecuados de bienestar psicológico en los adolescentes estudiantes, correlaciones significativas entre el bienestar y las fortalezas del carácter de valentía, perseverancia, gratitud, inteligencia social y autocontrol, además de algunas diferencias significativas en la dimensión aceptación del bienestar psicológico y en la fortaleza de la inteligencia emocional de acuerdo con el género en los estudiantes.

Palabras clave: Bienestar psicológico, fortalezas del carácter, adolescentes, secundaria. 
García-Álvarez et al. Promoción del bienestar psicológico en la secundaria

\section{ABSTRACT}

With the purpose of promoting psychological well-being in educational centers, a psychoeducational experience based on the cultivation of character strengths was designed for secondary school students. The research had the following objectives: to determine the level of psychological well-being in high school adolescents; To analyze the relationships between psychological well-being and character strengths; To establish if there are differences of gender and according to the educational center with respect to the psychological well-being - its dimensions - and the character strengths in the secondary school adolescents. Methodologically, it conforms to descriptive research with cross-sectional and correlational design through the collection of data through psychometric measures with adequate consistent and validity indexes. The results reveal adequate levels of psychological well-being in adolescents students, significant correlations between well-being and character strengths namely courage, perseverance, gratitude, social intelligence and self-control, in addition to some significant differences in the acceptability dimension of psychological well- being and in the strength of emotional intelligence, according to gender in students.

Keywords: Psychological well-being, character strengths, adolescents, high school.

\section{INTRODUCCIÓN}

La psicología positiva es la ciencia que se ocupa de estudiar el bienestar psicológico en la existencia humana (Seligman, 2011), busca investigar de forma controlada, seria y sistemáticamente el funcionamiento psicológico óptimo, es decir, el estudio de las cualidades, fortalezas y emociones positivas en los seres humanos; en efecto, se ha demostrado que las anteriores pueden actuar como elementos barreras o factores de protección ante eventos traumáticos, críticos de la vida o situaciones difíciles a lo largo del ciclo vital, además de que aumentan la capacidad de recuperación de las personas, (Garassini y Zavarce, 2010).

El núcleo teórico de esta investigación es el enfoque de la psicología positiva que considera el bienestar desde la integración de la dimensión hedónica (basada en las emociones positivas) y la dimensión eudaimónica (mediante el ejercicio virtuoso). En la actualidad, existen varios modelos teóricos de bienestar, entre ellos setienen: a) modelo PERMA de Seligman (2011) basado en la integración - cultivo de emociones positivas, vida comprometida, relaciones sociales satisfactorias, vida con sentido, y logro y cumplimento de metas; b) el modelo de bienestar de Ryff (1989; Ryff \& Singer, 2006), este es el asumido en este estudio debido a que tiene gran aplicación así como validación en países hispanohablantes.

En ese sentido, Ryff plantea que el bienestar psicológico se puede definir como el esfuerzo que hacen las personas para perfeccionarse a sí mismas y capitalizar sus recursos en la mejor versión de sí. Este modelo de bienestar psicológico está fundamentado en la idea del funcionamiento óptimo en las diferentes áreas de la vida desde una posición eudaimónica del bienestar, es decir, el bienestar no solo puede ser estudiado desde el componente hedonista de la búsqueda del placer y la evitación del dolor, sino que se toman en consideración aspectos como los recursos personales, el crecimiento personal, ambiente, la responsabilidad, entre otros elementos, desde un enfoque multidimensional que va más allá de asunción del bienestar como una estabilidad entre afectos negativos y positivos, (García y Soler, 2016). 
Desde este modelo se tienen que las dimensiones del bienestar son (Casullo y Castro, 2000; Aranguren e Irrazabal, 2015):

a) aceptación, elemento referido a una apreciación positiva de sí mismo, así como conocer - reconocer las cualidades positivas y negativas;

b) relaciones sociales, elemento relacionado con la esfera social en la calidad de relaciones y vínculos con los demás que sean sanas, cálidas y confiables con empatía y afectividad;

c) autonomía, que hace referencia a la autodeterminación de la persona que se ve expresada en la capacidad de resistir presiones;

d) proyectos, elemento relacionado con el sentido de vida, con la capacidad de la persona de plantearse objetivos, dirigiendo acciones para cumplirlos,

e) control ambiental, referido a la capacidad de control y competencia con la que la persona cuente, aproveche oportunidades y la capacidad de manejar el medio y la propia existencia; f) crecimiento personal, elemento muy importante que hace mención al sentimiento que va creciendo y desarrollando a lo largo del tiempo; se ve expresado en verse a sí mismo en progreso, y potenciar recursos personales para estar abiertos a nuevas experiencias y a cambiar de comportamiento.

En relación con las virtudes y fortalezas del carácter, son aquellos rasgos que Seligman (2002) denomina el buen carácter, haciendo referencia a la interacción del conjunto de rasgos positivos de la personalidad que se ven expresados en modos de pensar, actuar y sentir; estos son reconocidos como fortalezas por su valor en la plenitud, bienestar y promoción del desarrollo humano, así como por su valor moral en el sentido de que deben ser deseables e intrínsecas para vivir una vida virtuosa, deben generar gratificación, son duraderas, estables, medibles y además son ubicuas. Peterson y Seligman (2004) realizaron una clasificación de estas fortalezas mediante la consideración de seis virtudes humanas que contienen 24 fortalezas; en el apartado de la descripción del programa Creciendo Fuertes se describirán las tomadas en cuenta en el diseño de la investigación. Ahora bien, esta forma de concebir al hombre y su bienestar, trajo un gran impacto a nivel educativo debido a que desde antes se le exige a la educación el desarrollo integral de la personalidad de los educandos que cuenten con habilidades para la vida. En ese sentido, la psicología positiva ha tenido evidencias a nivel mundial sobre cómo incrementar el bienestar psicológico en las escuelas, como los programas Penn Resiliency Program, y el Strath Haven Positive Psychology Curriculum realizados por Seligman et al, (2009) basados en la promoción de las fortalezas del carácter y la resiliencia en niños y adolescentes con efectos demostrados en el aumento del bienestar.

Asimismo, el esfuerzo de Australia de realizar un currículo educativo basado en la psicología positiva dirigido por Seligman et al (2009). En Iberoamérica, Arguís et al, (2011) diseñaron un programa llamado Aulas Felices bajo la misión de promover el bienestar psicológico en estudiantes; como se puede apreciar se han llevado múltiples esfuerzos psico educativos centrados en la promoción del bienestar psicológico (salud mental) en centros educativos.

De allí que la justificación de esta investigación se basa en constituirse como una experiencia piloto de promoción del bienestar psicológico en centros educativos en Montevideo, que además generará datos empíricos sobre las variables bienestar psicológico y fortalezas del carácter en estudiantes adolescentes locales que sirvan como base para futuros estudios, conjuntamente de que se puedan desarrollar vías de intervención, y acciones educativas para la promoción de la salud mental. De igual manera, los resultados buscan aportar a otros 
estudios y experiencias científicas en las líneas de investigación sobre bienestar psicológico en ámbitos educativos formales.

Por todo lo expuesto anteriormente, se formulan las siguientes preguntas de investigación: ¿Cuáles serán las características de un programa basado en la promoción del bienestar psicológico?; ¿Cuál será el nivel de bienestar psicológico después de la aplicación del programa Creciendo Fuertes I?; ¿Cuáles serán las relaciones entre el bienestar psicológico y las fortalezas del carácter, a saber: autocontrol, curiosidad y amor por el aprendizaje, inteligencia social, valentía, perseverancia, humor y gratitud en los estudiantes adolescentes?;¿Existirán diferencias de género en cuanto al bienestar psicológico - sus dimensionesy a las fortalezas del carácter en los adolescentes de secundaria?; ¿Existirán diferencias en cuanto al bienestar psicológico - sus dimensiones- y a las fortalezas del carácter en los adolescentes de secundaria de acuerdo con el tipo de centro educativo?.

De modo que, los objetivos de esta investigación son: describir las características del programa Creciendo Fuertes I; determinar el nivel de bienestar psicológico en los adolescentes de secundaria luego de la aplicación del programa Creciendo Fuertes I; analizar las relaciones entre bienestar psicológico y las fortalezas del carácter: autocontrol, curiosidad y amor por el aprendizaje, inteligencia social, valentía, perseverancia, humor y gratitud en los estudiantes adolescentes; establecer si existen diferencias de género con respecto al bienestar psicológico sus dimensiones- $y$ las fortalezas del carácter en los adolescentes de secundaria; establecer si existen diferencias en el bienestar psicológico - sus dimensiones- y las fortalezas del carácter en los adolescentes de secundaria, de acuerdo con el tipo de centro educativo.

\section{METODOLOGÍA}

Diseño: El diseño de esta investigación se enmarca en una metodología no experimental de tipo transversal con dos grupos, (Hernández, Fernández y Baptista, 2008; Montero y León, 2002). Para lograr apreciar las características del programa Creciendo Fuertes $I$, basado en las fortalezas del carácter, se tomó una medida de bienestar psicológico solo después de la aplicación del programa en dos grupos de estudiantes ( $A$ y $B$ ), este hecho metodológico se justifica debido a que la presente investigación se realizó en el ambiente natural de los estudiantes de secundaria (grupos ya formados por las instituciones educativas), es decir, se tienen muestras no escogidas al azar por los investigadores, hechos que junto a otros, imposibilitaron llevar a cabo algún diseño de tipo experimental o cuasiexperimental.

De modo que, esta investigación piloto corresponde a un diseño no experimental, descriptivo transversal con dos grupos (A y B) y correlacional, pues se buscó identificar la fuerza, magnitud y significancia entre bienestar psicológico y el conjunto de las fortalezas del carácter del programa, además de establecer algunas comparaciones entre los grupos de acuerdo con el género y el tipo de institución.

Muestra: La muestra estuvo conformada por dos grupos independientes de dos instituciones educativas en Montevideo, con la finalidad de llevar a cabo la experiencia de la promoción del bienestar psicológico y fortalezas del carácter del programa Creciendo Fuertes $I$ en dos instituciones educativas distintas; se mantiene el diseño no experimental debido a que los grupos no tuvieron interacción alguna. El grupo A (institución de carácter no público) estuvo conformado por 30 estudiantes de secundaria. Por otro lado, el grupo $B$ (institución de carácter público) estuvo conformado por 42 . De modo que la 
muestra total de estudiantes estuvo conformada por 72 estudiantes (36 masculinos y 36 femeninos), con una media de edad de 15 años.

Instrumentos: Se elaboró una batería psicométrica conformada por datos socio - demográficos, más la escala BIEPS - J de bienestar psicológico para adolescentes de Casullo (2002), que mide el bienestar psicológico en las dimensiones de aceptación, vínculos, proyectos y control, cada una con adecuados índices de consistencia interna por el alfa de Cronbach. Así también se incluyeron las sub escalas de las fortalezas del carácter, consideradas en el diseño de investigación del Inventario de Fortalezas del Carácter de la versión adaptada y validada por Rojas (2010) con base en la traducción española de Vázquez y Hervás (2002) del Values in Action Institute (VIA) de Peterson y Seligman (2004), a saber: autocontrol, curiosidad y amor por el aprendizaje, inteligencia social, valentía, perseverancia, humor y gratitud. Por cuanto, estas sub escalas cuentan también con adecuados índices de consistencia interna en el alfa de Cronbach, lo que quiere decir que la batería diseñada está conformada por instrumentos psicológicos válidos y confiables para los fines de investigación.

Procedimiento: La recolección de datos se realizó mediante la colaboración de los talleristas que aplicaron la batería psicológica sobre bienestar psicológico y fortalezas del carácter, luego de la aplicación del programa Creciendo Fuertes I que tuvo una duración de 8 meses (abril noviembre). Los datos fueron procesados en el programa estadístico IBM SPSS Statistics 23.0 mediante estadísticas descriptivas, pruebas de distribución normal para tomar la decisión de aplicar análisis paramétricos o no paramétricos para establecer correlaciones, prueba de contraste de hipótesis correspondientes para la diferenciación por géneros y el tipo de centro educativo, asimismo el nivel de significación fue de 0.05
Sobre el programa Creciendo Fuertes I El programa Creciendo Fuertes $I$ de "Jóvenes Fuertes" está diseñado bajo intervenciones en modalidades de talleres vivenciales; los mismos fueron facilitados por dos talleristas fijas, quienes trabajaron con el mismo grupo cada 15 días, es decir con una frecuencia bimestral. La duración de cada taller fue de un módulo -2 horas clase- (80 minutos aproximadamente). Previo al comienzo del programa de talleres Creciendo Fuertes I, se les aplicó a todos los estudiantes el cuestionario VIA (Virtues in Action) Survey of Character Strengths dirigido a jovenes, de la Universidad de Pennsylvania para que cada uno descubra sus 5 fortalezas preponderantes. Disponible en https:// www.authentichappiness.sas.upenn. edu/es/testcenter.

Con respecto al primer taller, el mismo es de carácter introductorio, denominado "Me conozco" cuyo objetivo es reflexionar acerca de las distintas dimensiones del ser humano y la responsabilidad personal que cada uno tiene en dirigir su propia vida hacia el desarrollo pleno de cada una de estas dimensiones para lograr la plenitud.

Luego, comienzan los talleres relacionados con el conjunto de fortalezas seleccionadas, a saber: autocontrol, curiosidad y amor por el aprendizaje, inteligencia social, valentía, perseverancia, humor y gratitud. En los talleres de estas fortalezas, primeramente se define la fortaleza para que los estudiantes tengan una comprensión acabaday clara de la misma. Esto se hace por medio de dinámicas y juegos para que los adolescentes construyan su propio aprendizaje. Una vez que los estudiantes aprehenden lo que implica la fortaleza, se reflexiona sobre acciones concretas a través de las cuales se concretizaría la misma. De modo que, se reflexiona sobre los beneficios y consecuencias al incorporar la fortaleza en el comportamiento cotidiano; este conocimiento hace que 
la integración de la fortaleza sea algo que quiera ser alcanzado o logrado y, por tanto, se transforma en un objetivo para los jóvenes.

Los objetivos se hacen explícitos y conscientes durante cada taller, dando lugar a propósitos voluntarios por parte de los estudiantes; posteriormente son anotados en un cuaderno, para luego ser operacionalizados en acciones concretas para llevar a cabo durante la semana. El propósito final de estos talleres es la práctica de acciones que eventualmente serán transformadas en hábitos operativos adecuados para su desarrollo, integrados al carácter de los estudiantes.

Posteriormente al taller, se vuelve al centro educativo para hacer una actividad de reforzamiento o apoyo a los 15 días; allí se analiza cómo transcurrió la semana con respecto a los propósitos planteados y se profundiza acerca de la fortaleza. Se continúa trabajando durante el resto del mes sobre la misma fortaleza.

Ahora bien, el programa Creciendo Fuertes I, diseñado con las ocho fortalezas para el 2016, se llevó a cabo en ocho meses (abril a noviembre) y se asistió a los dos centros educativos cada quince días. Se trabajó una fortaleza por mes, siendo las mismas seleccionadas teniendo en cuenta el desarrollo evolutivo de los estudiantes. En ese sentido, los lugares utilizados para los talleres fueron tanto salones de clase como un salón multiuso. Este último, propicia el trabajo en la modalidad de taller, pues permite el movimiento de mobiliario además de contar con una pantalla gigante al frente del grupo. Todos los talleres fueron acompañados de una presentación tipo power point teniendo en cuenta las edades de los participantes, y por tanto, el tipo de recursos a utilizar para favorecer la atención. Algunos ejemplos son: recursos visuales, audiovisuales, audibles y electrónicos.
Se tomaron en cuenta a la hora de la planificación del taller y su presentación, los distintos estilos de aprendizaje. Por tal motivo se incluyeron en los distintos talleres, actividades que apuntaron a satisfacer los distintos estilos de aprendizaje. Se incluyó en todos los talleres algún extracto de película - canción también apuntando a los intereses de los estudiantes.

A cada estudiante se le entregó al comenzar el año, una carpeta que contenía un cuaderno con actividades relacionadas con las ocho fortalezas que se completarían durante el año, además aquí podrían hacer sus anotaciones. Esa carpeta permaneció en la institución educativa debido a que los estudiantes debieron hacerse responsables de su carpeta y asistir al taller con ese material. En la misma se incluyeron distintos tipos de actividades (crucigramas, espacios para dibujar, escribir o crear, definiciones incompletas, entre otros) tipo registro de lo trabajado en el taller.

Ahora bien, en el momento de iniciar cada taller se partió de los conocimientos previos que los estudiantes sabían acerca de la fortaleza; desde ese lugar y a través de distintas dinámicas se van realizando esfuerzos didácticos para que los estudiantes construyeran su propio conocimiento de la fortaleza. Otro punto a destacar, es que se busca implicar a los jóvenes en el tema, utilizando ejemplos que tuvieran relación con sus intereses y preocupaciones, es decir, aprendizaje contextualizado. Muchas actividades propuestas tuvieron como objetivo que relacionaran lo que aprendieron con sus experiencias en la vida diaria.

En ese sentido, se utilizaron distintas formas de agrupamiento como estrategia organizativa, pero en muchas instancias se recurrió al trabajo en grupo ya que el mismo ofrece múltiples ventajas además de oportunidades para comenzar a poner en práctica las fortalezas. Una gran parte de las dinámicas planteadas requirieron del 
trabajo en contextos de colaboración. Algunas veces el trabajo fue de reflexión individual; en estas instancias se les dio a los participantes la posibilidad de elegir si querían compartir o no sus ideas, debido a que muchas veces se trataron temas personales que podían llegar a ser movilizadores para quienes los vivieron.

Es importante destacar que al comienzo de cada uno de los ocho talleres se realizó un breve ejercicio de tres minutos de "mindfulness" para lograr la conciencia plena en el aquí y ahora, además para que los alumnos se focalizaran únicamente en el tema a tratar. Al finalizar cada taller se entregó a los participantes una hoja de evaluación en donde se recogió la opinión acerca del taller, las talleristas, película, modificaciones que le harían al taller, ideas centrales, etc.

Para comenzar a describir mes a mes el programa Creciendo Fuertes, en el primer mes, se realizó el primer taller en cual se hizo una breve introducción de lo que es la asociación Jóvenes Fuertes sobre su misión, visión y objetivos. En la misma instancia se trabajó con el taller - Me Conozco- en el cual se consideraron las distintas dimensiones del ser humano. A los quince días en la fase de apoyo, se retomó el tema de las distintas dimensiones y allí se profundizó acerca de la responsabilidad personal que cada uno tiene en dirigir su propia vida hacia el desarrollo pleno de cada una de estas dimensiones para lograr la plenitud.

En el segundo mes, se trabajó con la fortaleza de la valentía, cuya idea central fue no echarse atrás por el miedo, el desafío, los cambios, las dificultades o el dolor; defender lo que es justo a pesar de la oposición y actuar de acuerdo con las convicciones, aunque sean impopulares. Incluyó la valentía física pero no se limitó a esta. En la fase del apoyo a los quince días se trabajó la idea de los cambios y los miedos, muy pertinente de acuerdo con las tareas evolutivas de la adolescencia.
Durante el tercer mes, se trabajó la fortaleza de la inteligencia social. El objetivo fue que los estudiantes pudieran entender que dicha fortaleza implicaba ser consciente de los propios sentimientos y de los demás; también el conocimiento de uno mismo y de los demás, conocer cómo actuar en las situaciones sociales y cómo hacer que los otros se sientan bien. En la fase del apoyo se trabajó con la empatía. El objetivo fue intentar ponerse en el lugar del otro. Ayudar a comprender diferentes perspectivas de un problema o conflicto y fomentar en los adolescentes, actitudes de tolerancia y de respeto hacia las personas que tienen ideas o conductas diferentes a las suyas.

En el cuarto mes, se trabajó "la fortaleza del humor". El objetivo fue promover el disfrute por reír y hacer sonreír, así como alegrar a otros. En la fase del apoyo se trabajó con descatastrofizar las tragedias. En ese taller se fomentó el colocar las situaciones en perspectiva y centrarse en el momento presente.

En el quinto mes, se trabajó "la fortaleza de la curiosidad", el cometido del mismo fue interesarse por el mundo, aventurarse al conocimiento por cuenta propia, estar abierto a la experiencia, explorar y descubrir. Asimismo, se impulsó el deseo de conocer, mejorar y manejar nuevas habilidades. En la fase del apoyo se trabajó otra fortaleza: "amor por el aprendizaje", ya que ambas fortalezas están estrechamente vinculadas. Las ideas principales fueron disfrutar de aprender nuevos tópicos e incorporar nuevas habilidades además de sumar conocimientos a los ya previos de manera sistemática.

En el sexto mes, se trabajó con la "fortaleza del autocontrol". El objetivo fue que los estudiantes lograran regular lo que sienten y piensan; ser disciplinados; controlar los apetitos y necesidades, así como las propias emociones y necesidades. Durante la fase del apoyo se trabajó con: "¿Sé 
García-Álvarez et al. Promoción del bienestar psicológico en la secundaria

decir que no?”. Aquí se intentó descubrir estrategias para saber responder a las presiones a las cuales se ven enfrentados los adolescentes.

Durante el séptimo mes se trabajó con "la fortaleza de la gratitud". El objetivo fue que los estudiantes entendieran la gratitud como experiencia de ser consciente de agradecer las cosas buenas que suceden y tomarse el tiempo para celebrar la vida, aunque sea en pequeñeces. En el apoyo se trabajó "agradecer y celebrar.". Aquí se insistió acerca del desarrollo de las emociones positivas.

En el octavo mes, se trabajo con la "fortaleza de la perseverancia". Se buscó reflexionar acerca de la idea de acabar lo que se empieza; persistir en una acción a pesar de los obstáculos; concentrarse en lo que se hace y dedicar esfuerzo a conseguir objetivos y encontrar satisfacción en alcanzarlos. En la fase del apoyo se continuó con la perseverancia y distintas maneras de sobreponerse a las dificultades, de aplicar la imaginación de manera diferente cada vez, con el fin de resolver los problemas a los que se enfrentan.

\section{RESULTADOS}

Para comenzar a exponer los resultados encontrados, luego de la aplicación del programa "Creciendo Fuertes I", descrito en el apartado anterior, se tienen los resultados de bienestar psicológico para el grupo A y el grupo B (véase tabla 1). Se aprecia que ambos grupos de adolescentes estudiantes presenta un nivel adecuado de bienestar psicológico, presentaron percentil 50, es decir, la muestra de adolescentes está por encima del $50 \%$ de la población de referencia iberoamericana.

Ahora bien, para determinar el tipo de distribución de los datos en esta muestra, se realizó la prueba de " bondad
Tabla 1. Bienestar psicológico en adolescentes estudiantes según el grupo.

\begin{tabular}{|c|c|c|c|c|c|}
\hline \multicolumn{6}{|c|}{ Grupo A Institución de carácter no público } \\
\hline Variable & Participantes & Mínimo & Máximo & $\square$ & $\boldsymbol{\sigma}$ \\
\hline Control bienestar & 30 & 7 & 12 & 10,31 & 1,510 \\
\hline Vínculos bienestar & 30 & 6 & 9 & 8,40 & 0,914 \\
\hline Proyectos bienestar & 30 & 5 & 9 & 8,06 & 1,083 \\
\hline Aceptación bienestar & 30 & 3 & 9 & 7,51 & 1,522 \\
\hline Bienestar psicológico & 30 & 27 & 39 & 34,29 & 3,092 \\
\hline \multicolumn{6}{|c|}{ Grupo B Institución de carácter publico } \\
\hline Variable & Participantes & Mínimo & Máximo & $\square$ & $\boldsymbol{\sigma}$ \\
\hline Control bienestar & 42 & 5 & 12 & 10,83 & 1,685 \\
\hline Vínculos bienestar & 42 & 6 & 9 & 8,29 & ,908 \\
\hline Proyectos bienestar & 42 & 4 & 9 & 7,88 & 1,484 \\
\hline Aceptación bienestar & 42 & 5 & 9 & 7,87 & 0,900 \\
\hline Bienestar psicológico & 42 & 29 & 39 & 34,88 & 2,724 \\
\hline
\end{tabular}

de ajuste de Kolmogorov-Smirnov “, en la cual se obtuvo una significancia de 0.01 siendo menor a 0.05 , lo que se interpreta que la distribución de datos no asume la normalidad, de modo que se ejecutaron análisis no paramétricos para los siguientes resultados. En ese sentido, para determinar las correlaciones entre las variables de "bienestar psicológico" y las fortalezas, se empleó el coeficiente de correlación de Spearman, véase tabla 2.

De acuerdo con establecer si existen diferencias de género con respecto al bienestar psicológico -sus dimensionesy las fortalezas del carácter en los adolescentes de secundaria, los resultados obtenidos a través de la prueba de "contraste de hipótesis no paramétrica" de U de Mann-Whitney muestran que no hay diferencias significativas con respecto al bienestar psicológicos $(z=-1,638, p>0.05)$ ni en sus dimensiones: control $(z=-, 424$, $p>0.05)$, vínculos $(z=-, 865, p>0.05)$ $y$ proyectos $(z=-, 733, p>0.05)$ de acuerdo con el género. 
Tabla 2. Correlaciones de bienestar psicológico y las fortalezas en adolescentes estudiantes

\begin{tabular}{cccccccc}
\hline Bienestar psicológico & $\begin{array}{c}\text { Inteligencia } \\
\text { social }\end{array}$ & Autocontrol & $\begin{array}{c}\text { Amor por el } \\
\text { aprendizaje }\end{array}$ & Humor & Perseverancia & Gratitud & Valentía \\
\hline Rho de Spearman & $0,386^{* *}$ & $0,350^{* *}$ & 0,151 & 0,168 & $0,436^{* *}$ & $0,396^{* *}$ & $0,439^{* *}$ \\
$\begin{array}{l}* * \text { La correlación es significativa } \\
\text { en el nivel 0,01 (bilateral). }\end{array}$ & & & & & & & \\
\hline
\end{tabular}

Respecto a las fortalezas del carácter, tampoco se encontraron diferencias significativas de acuerdo con el género, a saber: autocontrol ( $z=--, 412, p>$ $0.05)$; amor por el aprendizaje $(z=-, 676$, $p>0.05)$; humor $(z=--, 152, p>0.05)$, perseverancia $(z=-, 271, p>0.05)$; gratitud $(z=-1,377, p>0.05)$ y valentía $(z=--, 028, p>0.05)$. Inversamente, sí se encontraron diferencias significativas de acuerdo con el género en la dimensión de aceptación del bienestar psicológico $(z=-, 3199, p<0.05)$, $y$ en la fortaleza de la inteligencia emocional $(z=-2,271$, $\mathrm{p}<0.05)$.

Siguiendo con establecer si existen diferencias en el bienestar psicológico y a las fortalezas del carácter en los adolescentes de secundaria de acuerdo con el tipo de centro educativo, los resultados obtenidos a través de la prueba de "contraste de hipótesis no paramétrica" de U de Mann-Whitney muestran que no hay diferencias significativas de acuerdo con bienestar psicológico $(z=-, 310, p>0.05)$ y en ninguna de sus dimensiones: control $(z=-1,262, p>0.05)$; vínculos $(z=-$ $1,269, p>0.05)$; proyectos $(z=-, 652$, $\mathrm{p}>0.05)$ y aceptación $(\mathrm{z}=-, 018, \mathrm{p}>$ $0.05)$.

Asimismo, los resultados tampoco arrojaron diferencias significativas en las fortalezas del carácter cultivadas en los estudiantes adolescentes de acuerdo al tipo de institución educativa: inteligencia social $(z=-1,421, p>$ $0.05)$; autocontrol $(z=-, 343, p>$ $0.05)$; amor por el aprendizaje $(z=-$ ,686, p > 0.05); humor ( $z=-, 063, p$ $>0.05)$; perseverancia $(z=-1,939$, $p>0.05)$ y valentía $(z=-1,136, p>$
0.05). Sin embargo, si se encontraron diferencias significativas en la fortaleza de la gratitud $(z=-2,100, p<0.05)$, en los adolescentes de acuerdo al tipo de institución (de carácter público / no público).

De manera adicional se realizó un análisis mediante la prueba de KruskalWallis para conocer si había diferencias significativas con la categoría de grupo basada en los niveles de bienestar (alto, medio y bajo), arrojó que no hay diferencias en los niveles de bienestar.

\section{DISCUSIÓN}

Como se pudo apreciar, el nivel de bienestar psicológico en los grupos A y B se interpreta como adecuado, según Casullo (2002), estos estudiantes adolescentes presentan adecuada sensación de control y de competencia en sus habilidades personales que les permiten operar en el contexto para modificarlo con base en sus necesidades, asimismo presentan indicadores de autonomía, asertividad y toma de decisiones con algún grado de independencia lógica para la etapa de la adolescencia.

Estos resultados se interpretan en que los estudiantes cuentan con una visión de futuro que les permite llevar un proyecto de vida de acuerdo con sus valores, es decir, sienten que su vida tiene sentido. De igual manera, estos estudiantes muestran indicadores de adecuada aceptación de sí mismos, entonces se cree que pueden reconocer sus múltiples facetas y aceptar el pasado como parte de la existencia, 
y contar con vínculos personales con sus pares satisfactorios que generan bienestar. Vale a acotar, que estos resultados son similares a los expuestos por García y Soler (2016) en una muestra de adolescentes estudiantes de instituciones educativas en Montevideo.

Siguiendo con el análisis de las correlaciones encontradas entre el bienestar psicológico y las fortalezas del carácter, se tienen resultados bastantes congruentes con la literatura científica, en efecto, enlistando en orden decreciente las fortalezas con mayor correlación significativa con el bienestar en la presente investigación, se tienen: valentía, perseverancia, gratitud, inteligencia social y autocontrol.

Asimismo, varias investigaciones a nivel mundial han puesto de manifiesto las relaciones positivas - e incluso predictivas- con el bienestar psicológico en la adolescencia y adultez (Proyer, Ruch, \& Buschor, 2012; Park \& Peterson, 2009; Brdar \& Kashdan, 2010; Ruch et al., 2007; Peterson et al., 2007; Shimai et al., 2006; Park, Peterson, \& Seligman, 2004). Los resultados son congruentes además con los presentados por Sol Grinhauz (2012), García y Soler (2016); Park, Peterson \& Seligman (2004); García, Serrano y Pérez (2015) quienes reportaron correlaciones significativas de las fortalezas de valentía, perseverancia, gratitud, inteligencia social y autocontrol con el bienestar psicológico.

Por su parte, Martinez-Marti \& Ruch, (2014) publicaron que las fortalezas que más se relacionan con el bienestar son: inteligencia social y perseverancia; en otra instancia Buschor, Proyer, \& Ruch, (2013) evidenciaron que la curiosidad, amor por el conocimiento y la gratitud tienen fuerte impacto en el bienestar psicológico; Proyer et al. (2011) han informado fuertes correlaciones del humor con el bienestar psicológico; así como Park et al. (2004) demostraron que la gratitud es una de las fortalezas más valiosas en la varianza del bienestar.
Aunado, se ha encontrado en antecedentes que las fortalezas de la perseverancia y gratitud, al igual que las fortalezas cognitivas como la curiosidad y amor por el aprendizaje, tienen fuerte relación con la satisfacción vital en la adolescencia (Gimenez, 2010; Ruch et al. 2014).

Desde el punto de vista el teórico científico los resultados son congruentes con los postulados de la psicología positiva que explican que las fortalezas del carácter son rasgos de las virtudes humanas que las personas ponen en práctica en su vida cotidiana para cultivar el bienestar (Peterson y Seligman, 2004; Seligman, 2011), es decir, las fortalezas de la gratitud y la inteligencia social permiten a los adolescentes manejarse de manera adecuada en el mundo social relacionándose con los componentes de vínculos sociales satisfactorios y aceptación personal del bienestar psicológico.

En tal sentido, la valentía, el autocontrol y la perseverancia son fortalezas que pueden ayudar a los adolescentes en situaciones específicas para alcanzar objetivos, considerando los obstáculos que puedan originarse en el camino, relacionándose con las dimensiones del bienestar psicológico de proyectos de vida y control ambiental a través de la perseverancia, y la dimensión de la aceptación personal necesita de la fortaleza de la valentía para poder reconocerse y plantear posiciones, (Ryff, 1989; García y Soler, 2016).

Por otra parte, la literatura científica del estudio de las fortalezas del carácter, de acuerdo con el género, manifiesta que existen diferenciassignificativas en cierto grupo de fortalezas, a saber: aquellas fortalezas de orden interpersonales favorecen a las mujeres, mientras que para los hombres existe mayor relación en las fortalezas intelectuales, (Sánchez - Hernández, 2015). Asimismo, diversos autores han puesto de manifiesto las 
diferencias significativas de acuerdo con el género en la fortaleza de la inteligencia social, (Linley et al., 2007; Ovejero y Cardenal, 2015; Gimenez, 2010; Toner et al. 2012; Ovejero, 2010; Sol Grinhauz, 2015) explican que probablemente las diferencias encontradas se deban a la conformidad de las normas convencionales de género, en que las mujeres están más orientadas culturalmente al manejo de aspectos socio emocionales que los hombres.

Ahora bien, según Peterson \& Seligman (2004) las fortalezas del carácter han sido estudiadas en relación con las variables socio - demográficas de manera modesta, en la presente investigación se encontró diferencia significativa en la fortaleza de la gratitud de acuerdo con el tipo de centro educativo (institución de carácter público / no público), sabiendo que la gratitud es una fortaleza de la virtud de la trascendencia, que consiste en reconocer aquello que sí se tiene, sobre aquello que no, implica dar gracias por cosas, esfuerzos y voluntades de los demás, y estar agradecido de las cosas buenas de la vida que no necesariamente se deben tener. Es compresible hallar estos resultados, pues uno de los centros educativos es de carácter público con ciertas condiciones de contexto crítico. En efecto, Shimai et al. (2006) exponen que las variables socio - demográficas pueden influir en la relación de fortalezas del carácter y salud.

Con respecto a las diferencias no significativas del bienestar psicológico de acuerdo al género en los adolescentes estudiantes del presente estudio, la literatura científica no presenta evidencia empírica suficiente sobre diferencias significativas en bienestar psicológico en hombres y mujeres desde el modelo de Ryff (1989); es más, existen resultados contradictorios; en algunos casos las mujeres obtienen puntajes mayores o viceversa.
Sin embargo, los resultados aquí encontrados son congruentes con los expuestos por García y Soler (2016) y Visani et al. (2011) quienes no encontraron diferencia de acuerdo con el género, en determinados casos las mujeres presentan diferencias en algunas de las dimensiones del bienestar sobre los hombres (García - Alandete, 2013; Zubieta, Muratori y Fernández, 2012; Del Valle, Hormaechea y Urquijo, 2015), en este caso existe diferencia a la dimensión de aceptación de bienestar psicológico de acuerdo con el género.

\section{CONCLUSIONES}

En definitiva, este trabajo de investigación concluye de acuerdo con sus objetivos: a) niveles adecuados de bienestar psicológico en adolescentes estudiantes; b) correlaciones significativas entre el bienestar psicológico y las fortalezas del carácter: valentía, perseverancia, gratitud, inteligencia social y autocontrol; c) diferencia significativa de acuerdo con el género en la fortaleza de la inteligencia social y, en la dimensión aceptación del bienestar psicológico en el grupo de adolescentes estudiantes; d) diferencia significativa solo en la fortaleza de la gratitud en los adolescentes estudiantes de acuerdo con el tipo de centro educativo (de carácter público / no público).

Estas conclusiones deben tomarse con cautela, pues en ningún momento se busca su generalización para otras muestras uruguayas adolescentes, ni se pretende probar la efectividad del programa descrito, debido a que desde las limitaciones de este estudio se tienen factores situacionales, una muestra mediana y una metodología transversal que solo permitió recoger datos de la medida de bienestar después de la aplicación del programa Creciendo Fuertes I, es decir, no se tenían datos de medidas pre y post del programa. En ese sentido, el principal aporte de este 
trabajo es describir a nivel operacional didáctico un programa basado en ocho fortalezas para ser implementado en instituciones educativas con la mira de generar factores de protección personal sobre el bienestar psicológico de los estudiantes ante factores y conductas de riesgo, (García y Serrano, 2017). De modo que, esta experiencia de investigación, con la finalidad de la promoción del bienestar psicológico en la secundaria, se configura como un pequeño grano de arena para ayudar a formar un movimiento científico educativo que busque en sus propósitos el desarrollo pleno de los estudiantes en Uruguay.
Desde estas limitaciones, se recomienda para futuras investigaciones tomar muestras más amplias; al ser una experiencia piloto su principal limitación es la muestra reducida. De igual manera, se recomienda incluir otras variables de psicología positiva y de la realidad educativa, a saber: rendimiento académico, rasgos de personalidad y otras fortalezas del carácter. Así también sería interesante llevar a cabo investigaciones longitudinales con diseños cuasi experimentales para probar la efectividad del programa Creciendo Fuertes I o de otros de programas similares que promuevan el bienestar psicológico en las escuelas con estudiantes y docentes, como es el caso de la organización Jóvenes Fuertes en Uruguay, (véase: http://www. jovenesfuertes.org/inicio/es).

\section{REFERENCIAS}

Aranguren, M., y Irrazabal, N. (2015). Estudio de las propiedades psicométricas de las escalas de bienestar psicológico de Ryff en una muestra de estudiantes argentinos. Ciencias Psicológicas, 9(1), 73-83.

Arguís, R., Bolsas, A. P., Hernández, S. y Salvador, M. (2010). Programa Aulas Felices. Psicología Positiva aplicada a la educación. Recuperado de http:// catedu.es/psicologiapositiva/

Brdar, I., \& Kashdan, T.B. (2010). Character strengths and well-being in Croatia: An empirical investigation of structure and correlates. Journal of Research in Personality, 44, 151-154.

Buschor, C., Proyer, R. T., \& Ruch, W. (2013). Self- and peer-rated character strengths: How do they relate to satisfaction with life and orientations to happiness? Journal of Positive Psychology, 8 (2), 116-127.
Casullo M. \& Castro Solano, A. (2002). Patrones de personalidad, síndromes clínicos y bienestar psicológico en adolescentes. Revista de Psicopatología y Psicología Clínica, $7(2), 129-140$.

Casullo, M. (2002). Evaluación del BienestarPsicológicoen Iberoamérica. Buenos Aires: Paidós.

Del Valle, M., Hormaechea, F., y Urquijo, S. (2015). El Bienestar Psicológico: Diferencias según sexo en estudiantes universitarios y diferencias con población general. Revista Argentina de Ciencias del Comportamiento, 7(3), 00. Recuperado en 28 de febrero de 2017, de http://www.scielo.org.ar/scielo. php?script $=$ sci arttext\&pid=S1852$42062015000300003 \& \operatorname{lng}=e s \& t \operatorname{lng}=$ es. 
Garassini, M. y Zavarce, P. (2010). Historia y marco conceptual de la Psicología positiva. En Garassini, M. y Camili, C. (Comp.) Psicología positiva: estudios en Venezuela. Venezuela: Monfort.

García, D. y Serrano, M. (2017). La inteligencia emocional: capacidad para el desarrollo humano en el marco de la orientación educativa. Revista Convocación 30, VII , 56-67

García, D. y Soler, M. (2016). Bienestar psicológico en la secundaria: estudio piloto con docentes y adolescentes. Revista Convocación, 29, IX, 34-44

García, D.; Serrano, M. y Pérez, C. (2015). Bienestar psicológico e inteligencia emocional con relación a la humanidad y amor en estudiantes de educación integral. Encuentro Educacional, 22 (2), 153-164.

García-Andalete, J. (2013). Bienestar psicológico, edad y género en universitarios españoles. Salud \& Sociedad, 4(1), 48-58.

Giménez, M. (2010). La medida de las fortalezas psicológicas en adolescentes (VIA- youth), relación con clima familiar, psicopatología y bienestar psicológico. Tesis Doctoral no publicada. Facultad de Psicología, Universidad Complutense de Madrid.

Hernández, R., Fernández, C. y Baptista, P. (2008). Metodología de la investigación científica. México: Mc Graw Hill.

Linley, A., Maltby, J., Wood, A. M., Joseph, S., Harrington, S., Peterson, C., et al. (2007). Character strengths in the United Kingdom: The VIA Inventory of Strengths. Personality and Individual Differences, 43(2), 341-351, doi: $10.1016 / j$. paid.2006.12.004

Martinez-Marti, M. L., \& Ruch, W. (2014). Character strengths and well-being across the life span: data from a representative sample of German-speaking adults living in Switzerland. Frontiers in Psychology, 5, 1253. doi: 10.3389/ fpsyg.2014.01253

Montero, I. y León, O. (2002). Clasificación y descripción de las metodologías de investigación en Psicología. Revista Internacional de Psicología Clínica y de la Salud/Internacional Journal of Clinical and Health Psychology, 2, 503-508.

Ovejero M., \& Cardenal, C. (2015). "Las fortalezas humanas desde la perspectiva de género: un estudio exploratorio en población española". Revista Mexicana de Investigación en Psicología, 7(2), 72-92

Ovejero, M. (2010). Las fortalezas humanas desde la perspectiva de género. Trabajo de fin de master. España: Universidad Complutense.

Park, N., \& Peterson, C. (2009b). Strengths of character in schools. In R. Gilman, E. S. Huebner, \& M. J. Furlong (Eds.), Handbook of positive psychology in schools (pp. 65-76). New York: Routledge

Park, N., Peterson, C., \& Seligman, M. E. P. (2004). Strengths of character and well-being. Journal of Social \& Clinical Psychology, 23, 603-619. Peterson et al., 2007

Peterson, C. \& Seligman, E. P. (2004). Character strengths and virtues: a handbook and classification. Oxford: Oxford University Press y American Psychological Association.

Proyer, R. T., Gander, F., Wyss, T., \& Ruch, W. (2011). The relation of character strengths to past, present, and future life satisfaction among german-speaking women. Applied Psychology: Health and Well-Being, 3 (3), 370-384. 
Proyer, R. T., Ruch, W., \& Buschor, C. (2012). Testing strengths-based interventions: A preliminary study on the effectiveness of a program targeting curiosity, gratitude, hope, humor, and zest for enhancing life satisfaction. Journal of Happiness Studies.

Rojas, R. (2010). "Fortalezas del carácter, variables sociodemográficas y salud en trabajadores de sector público". [Trabajo de tesis Magister en Psicología]. Caracas- Venezuela: Universidad Simón Bolívar.

Ruch, W., Huber, A., Beermann, U., \& Proyer, R. T. (2007). Character strengths as predictors of the "good life" in Austria, Germany and Switzerland. In Romanian Academy, "George Barit" Institute of History, Department of Social Research (Ed.), Studies and researches in social sciences (Vol. 16). Cluj-Napoca, Romania: Argonaut Press, 123-131.

Ruch, W., Weber, M., Park, N., y Peterson, C. (2014). Character strengths in children and adolescents: Reliability and initial validity of the german values in action inventory of strengths for youth (german VIA-youth). European Journal of Psychological Assessment, 30(1), 57-64

Ryff, C. D. (1989). Happiness is everything, or is it? explorations on the meaning of psychological well-being. Journal of Personality \& Social Psychology, 57(6), 1069-1081

Ryff, C. D., \& Singer, B. H. (2006). Best news yet on the six-factor model of well-being. Social Science Research, 35, 1103-1119. DOI: 10.1016/j. ssresearch.2006.01.002

Sánchez-Hernández, Ó. (2015). Sobre las fortalezas humanas y las diferencias de género. Revista Mexicana de Investigación en Psicología, 7(2), 107-116
Seligman, M. (2011). Flourish: a visionary new understanding of happiness and well-being. New York: Free Press

Seligman, M. (2002). La auténtica felicidad. Barcelona: Grijalbo.

Seligman, M., Ernst, R., Gillham, J., Reivich, K. y Linkins, M. (2009). Positive education: positive psychology and classroom interventions. Oxford Review of Education, 35(3), 293-311.

Shimai, S., Otake, K., Park, N., Peterson, C., \& Seligman, M. E. P. (2006). Convergence of character strengths in american and japanese young adults. Journal of Happiness Studies, 7, 311-322.

Sol Grinhauz, A. (2012). El análisis de las virtudes y fortalezas en niños: una breve revisión teórica. Psicodebate, $12,39-61$

Sol Grinhauz, A. (2015). El estudio de las fortalezas del carácter en niños: Relaciones con el bienestar psicológico, la deseabilidad social y la personalidad. Psicodebate, 15(1): 43-68

Toner, E., Haslam, N., Robinson, J., \& Williams, P. (2012). Character strengths and wellbeing in adolescence: Structure and correlates of the values in action inventory of strengths for children. Personality and Individual Differences, 52(5):637642

Visani, D., Albieri, E., Offidani, E., Ottolini, F., Tomba, E. y Ruini, C. (2011). Gender differences in psychological well-being and distress during adolescence. En I. Brdar (Ed.), The human pursuit of well-being: $A$ cultural approach (pp. 65-70). New York, NY: Springer.

Zubieta, M., Muratori, M. y Fernández, D. (2012). Bienestar subjetivo y psicosocial: explorando diferencias de género. Salud \& Sociedad, 3(1):66-76. 